\title{
Efficient storage of urodynamic signals by computer: application of FAN adaptive sampling
}

\author{
R. van Mastrigt ${ }^{1}$ and D. J. Griffiths ${ }^{1,2}$ \\ Departments of ${ }^{1}$ Urology and ${ }^{2}$ Biomedical Physics and Technology, Erasmus University, Rotterdam, The Netherlands
}

Accepted: May 30, 1988

Summary. Digital storage of urodynamic signals such as detrusor pressure and flowrate at a sufficiently high sampling rate (10 samples per second) to allow subsequent analysis requires considerable computer memory. A procedure for compressing these data by deleting redundant samples (the fan method of adaptive sampling) was tested. The method allows a flexible adaptation to specific hardware and a compromise between storage requirements and accuracy. In this study the number of samples required for adequate reconstruction of the detrusor pressure signal could be varied from $80 \%$ to $4 \%$ of the original number of samples by varying the average difference between reconstructed and original signal from 0.01 to $2 \mathrm{~cm} \mathrm{H}_{2} \mathrm{O}$. Fast components of the measurements (for example cough peaks) which were lost if a lower sampling rate or averaging was used to obtain equally low storage requirements were unaffected by this compression technique.

Key words: Adaptive sampling - Sample rate - Computer - Storage - Urodynamic signals - Flow - Detrusor pressure

\section{Introduction}

Urodynamic signals are being digitized and stored using computers so that they can be subsequently retrieved and analysed [8]. This process involves sampling the signal values at equidistant time intervals. The sampling rate must be sufficiently high for rapid changes to be adequately represented. For pressure and flow signals a rate of at least 10 samples per second is required. Consequently, the storage of all measured signals for a complete cystometry procedure requires a considerable storage facility. For example the storage of two signals (destrusor pressure and flow) digitized with a 16 bit digitizer at 10 samples per second during a half hour period requires $72 \mathrm{kByte}$ of computer memory. Although it would technically be possible to store such amounts of data for a significant number of patients, many restraints cause urodynamic computer users and programmers to refrain from doing so. Instead either lower sample rates are being used, or only those parts of the cystometry curves that are believed to be of interest are being stored $[5,6]$. The first solution causes significant distortion of fast events (e.g. coughs, the onset of micturition). The second requires either manual or automatic selection of parts of the curve that are of interest, which takes time and may act as a potential source of error. An adaptive sampling method may provide a more favourable alternative. Such a method examines each sample as it is taken and decides whether it is necessary for adequate reconstruction of the signal or not. In the latter case it is discarded. In fact, in urodynamic signals rapid changes which require a high sample rate often alternate with quiet intervals which might be sampled at much lower rates. The FAN is a computer algorithm for such adaptive sampling, first described in 1964 [3, 4]. It was shown to be superior to a number of other methods when applied to intracellular and extracellular cardiac potentials and electrocardiograms $[1,2,7]$. As the performance of adaptive sampling methods depends on the properties of the signals to which they are applied, the present study investigates the applicability of the FAN to urodynamic signals. To this end it was repeatedly applied to a series of equidistantly sampled and stored pressure and flowrate signals so that parameters controlling the adaptive sampling process could be varied.

\section{Methods}

In addition to the storage and subsequent analysis of selected signal parts in terms of contractility and urethral resistance using the 


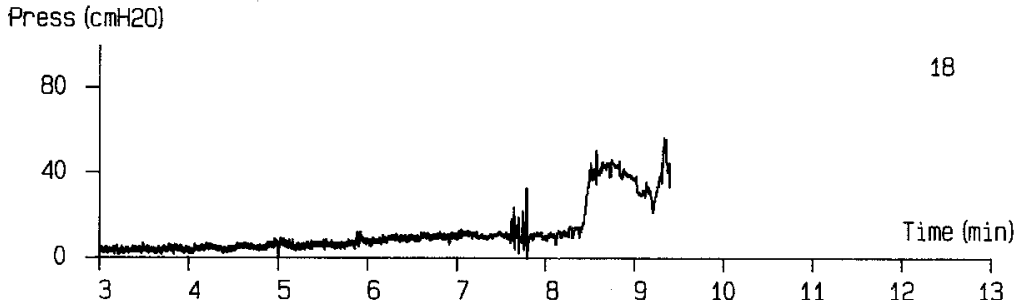

Flow (ml/s)
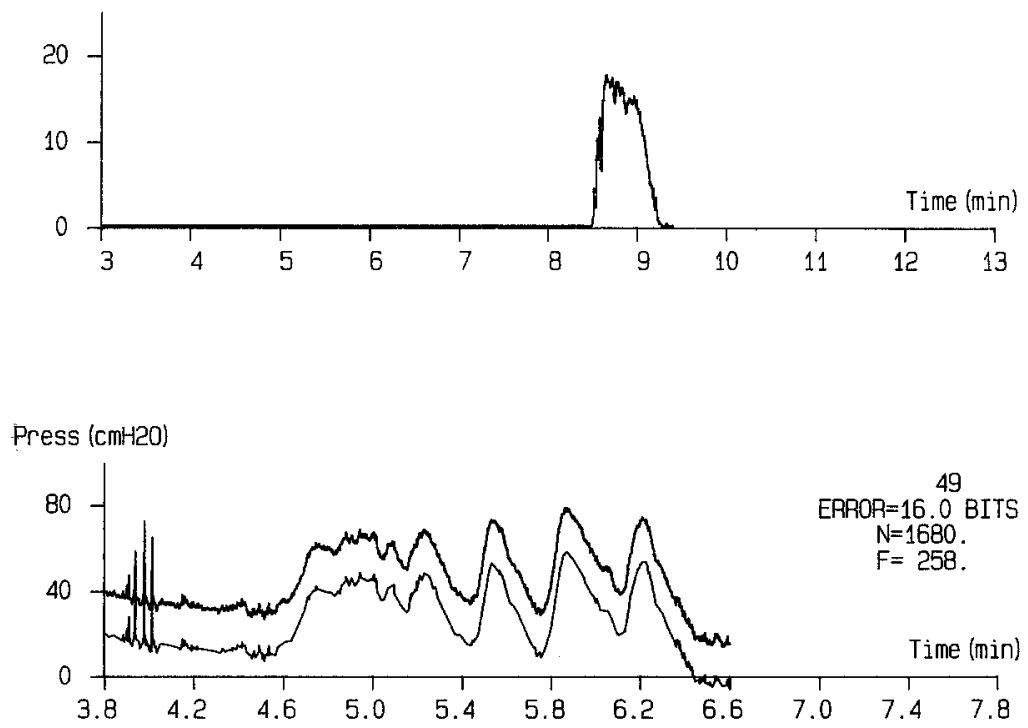

Fig. 1. Pressure and flowrate as stored at a rate of 10 samples per second during cystometry

Fig. 2. Partial pressure recording in greater detail. Upper trace represents original data, lower trace was reconstructed from the compressed version of the signal

computer program CLIM [6], detrusor pressure and flowrate from a mixed group of adult patients were stored directly on magnetic disc using a Digital Equipment Professional Computer cluster during routine cystometry procedures involving fluid filled transurethral catheters and standing voiding in an DISA flowmeter. From both signals 10 samples per second were stored during a maximum duration of $37.5 \mathrm{~min}$ per measurement. The signals were stored in a database structure for easy retrieval. A twelve bit bipolar A/D converter (thus giving output values ranging from -2047 to +2047 integer) at a full range of $200 \mathrm{~cm} \mathrm{H}_{2} \mathrm{O}$ for detrusor pressure and 50 $\mathrm{ml} / \mathrm{s}$ for flow was used. The smallest detectable change in the digitized signals therefore amounted to approximately $0.1 \mathrm{~cm} \mathrm{H}_{2} \mathrm{O}$ or $0.025 \mathrm{ml} / \mathrm{s}$.

A separate Fortran program was written which calculated a compressed version of each stored measurement using the fan algorithm. This was based on a linear extrapolation principle: if a given sample lay within a specified error from a straight line drawn through the two previous samples, it was considered redundant and discarded; otherwise it was stored. The original description of the algorithm [4] included an elegant and efficient method of implementing this decision procedure. Each measurement was compressed a number of times using different values of the maximum allowable error. From each compressed measurement a reconstructed version was calculated by linear interpolation between stored samples, and this was compared to the original measurement. Both the average difference between reconstructed and original measurement and the maximum difference were recorded for subsequent statistical analysis. Also the average and maximum intervals between successive samples from the compressed measurement and the number of samples in it in relation to that in the original measurement were stored. Statistical analysis was performed using the SPSS package.

\section{Results}

Figure 1 shows an example of a pressure and flow recording. 65 detrusor pressure recordings with an average duration of $10.3 \mathrm{~min}$ (range 4.5-26 $\mathrm{min}$ ) and 48 flow recordings with average duration of $8.9 \mathrm{~min}$ (range 4.5-16.5 $\mathrm{min}$ ) were separately processed. No preprocessing of the flow recordings was performed, so that these contained large stretches of base level noise during periods without flow. However "empty" flow recordings of measurements without voiding or leakage were excluded, which accounted for the difference between the numbers of detrusor pressure and flow recordings.

Figure 2 shows a partial pressure recording in greater detail. The upper trace represents the original data. Notice the four cough peaks at 4.0 min which resulted from an imperfect subtraction of bladder and abdominal pressure. The lower trace was reconstructed from the compressed version of the signal. In this case a maximum difference between reconstructed and original signal of 16 integer units (approximately equal to $1.6 \mathrm{~cm} \mathrm{H}_{2} \mathrm{O}$ ) was specified, which resulted in a reduction in the number of samples from 1680 to 258 . In all processed measurements the maximum error specified a priori in the fan compression algorithm was identical 


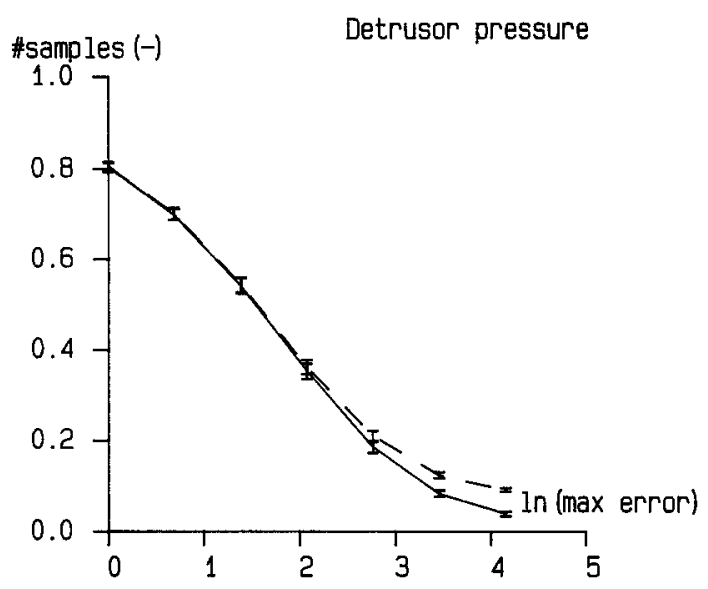

Fig. 3. Number of samples in fan-compressed detrusor pressure measurements as a function of maximum permitted error or difference between reconstructed and original signal. Average plus and minus standard error of the mean are shown. Solid line represents unmodified fan procedure, broken line represents results from procedure with interval limited to 16 samples maximum (see Discussion)

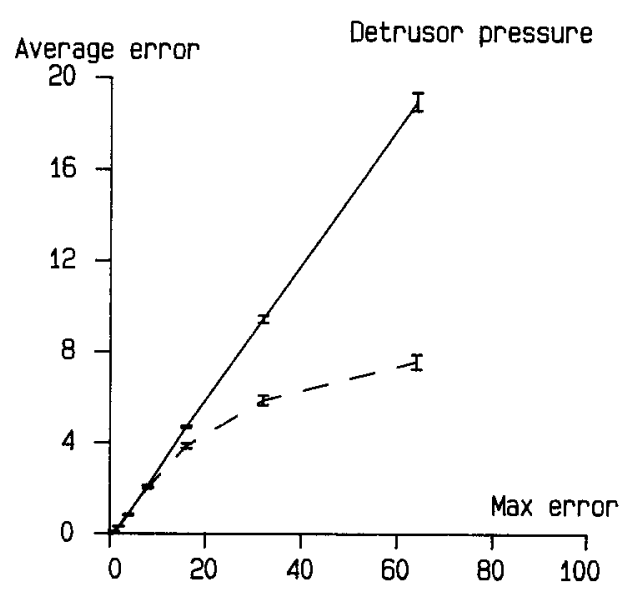

Fig. 4. The (absolute) average error or difference between reconstructed and original detrusor pressure signal as a function of the maximum permitted error. Solid line represents average plus and minus standard error of the mean resulting from the unmodified fan procedure, broken line represents results from procedure with interval limited to 16 samples maximum (see Discussion)

to the maximum difference calculated a posteriori between reconstructed and original signal. In Fig. 3 the drawn line shows for all 65 detrusor pressure curves the average of the number of samples in the compressed signal relative to the number in the original signal as a function of the logarithm of the maximum permitted error (in integer units). As the maximum permitted error was varied from 1 to 64 integer units $(0.1$ to $6.4 \mathrm{~cm}$ $\mathrm{H}_{2} \mathrm{O}$ ) the number of samples in the compressed signal varied from $80 \%$ to $4 \%$ of the number of samples in the

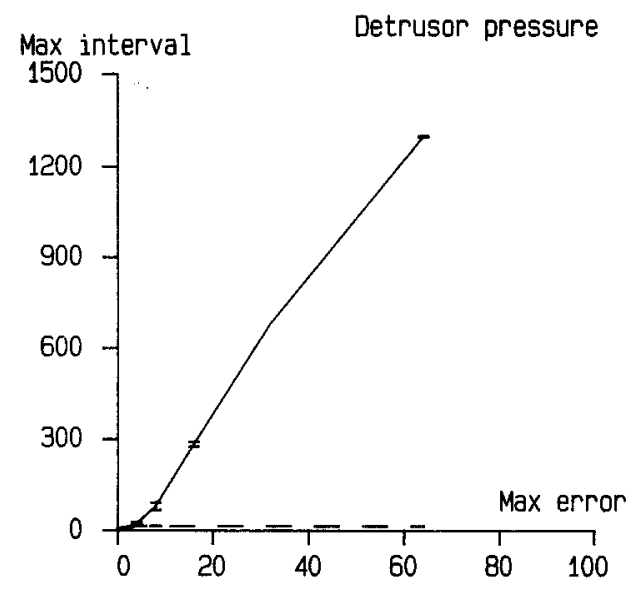

Fig. 5. The maximum number of contiguous samples discarded in each pressure measurement as a function of maximum permitted error. Solid line represents average plus and minus standard error of the mean resulting from the unmodified fan procedure, broken line represents results from procedure with interval limited to 16 samples maximum (see Discussion)

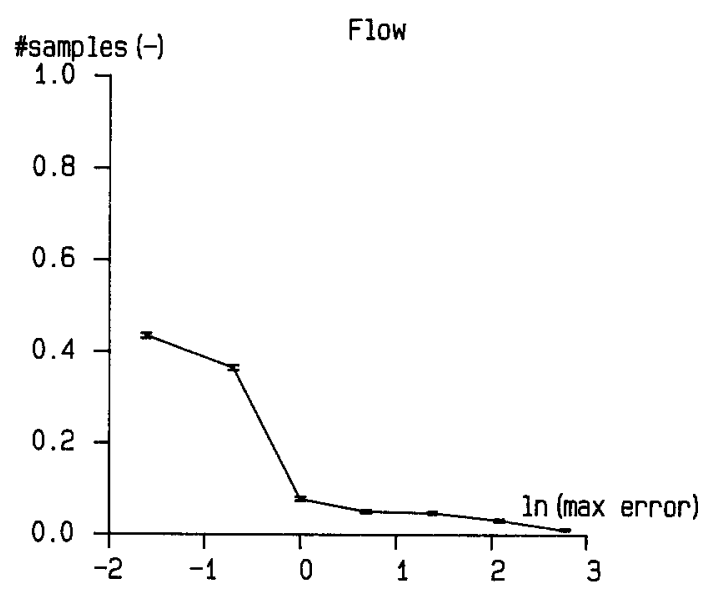

Fig. 6. Number of samples in fan-compressed flow measurements as a function of maximum permitted error or difference between reconstructed and original signal. Average plus and minus standard error of the mean are shown

original signal. At a maximum error of 16 integer units $\left(1.6 \mathrm{~cm} \mathrm{H} \mathrm{H}_{2} \mathrm{O}\right.$, ln (max error) $\left.=2.8\right)$ the number of samples was reduced to an average of $20 \%$, so that the effective average sample rate of the compressed signal was $0.2 * 10=2$ samples per second.

In Fig. 4 the solid line shows the almost linear relationship between the maximum permitted error and the average (absolute) difference between reconstructed and original detrusor pressure signals. For a maximum permitted error of 16 integer units (approx. 


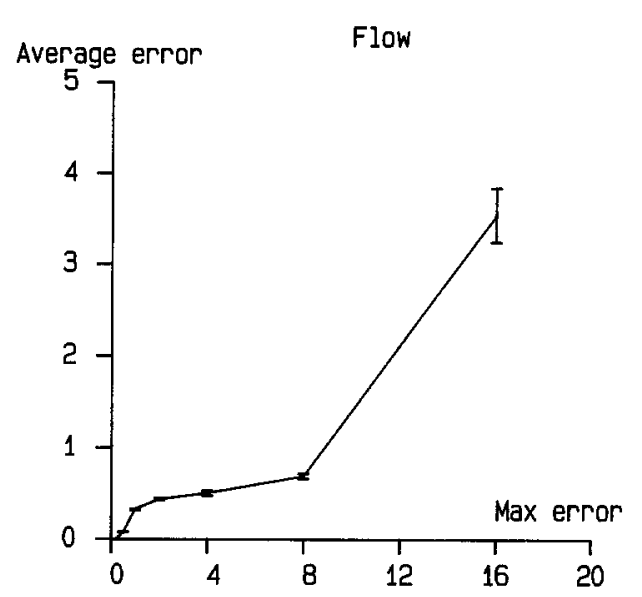

Fig. 7. The (absolute) average error or difference between reconstructed and original flow signal as a function of the maximum permitted error. Average plus and minus standard error of the mean are shown

$1.6 \mathrm{~cm} \mathrm{H}_{2} \mathrm{O}$ ) this average absolute difference was approximately 5 integer units (equal to $0.5 \mathrm{~cm} \mathrm{H}_{2} \mathrm{O}$ ).

The maximum number of contiguous samples discarded in each pressure measurement is depicted in Fig. 5 as a function of maximum permitted error. The solid line shows that at a maximum error of 16 integer units stretches of up to 300 consecutive samples, i.e. 0.5 min of signal, were discarded as redundant.

Figs. 6-8 shows the same graphs as Figs. 3, 4 and 5 for the flow measurements. With a maximum permitted error of 1 integer unit, equivalent to $0.025 \mathrm{ml} / \mathrm{s}$, flow measurements were compressed to $8 \%$ of the original number of samples. With larger permitted maximum errors the relation between average and maximum error (Fig. 7) was comparable to that found for detrusor pressure measurements, but at small maximum errors the average error was more constant and of the order of 0.5 integer units or $0.0125 \mathrm{ml} / \mathrm{s}$. The maximum number of consecutive redundant samples (Fig. 8) showed a plateau at approximately 4,000 samples or $6.6 \mathrm{~min}$ reflecting the average period during which no flow occurred in the measurements.

\section{Discussion}

Flow and detrusor pressure signals throughout filling and voiding cystometry, as depicted in Fig. 1 are distinctly different in properties relevant to data compression. Whereas the pressure signal consists of a continuously varying signal with considerable noise, the major part of the flow signal is formed by a recording of the zero baseline level and its small perturbations. The differences between these signals are reflected in the performance of the fan data

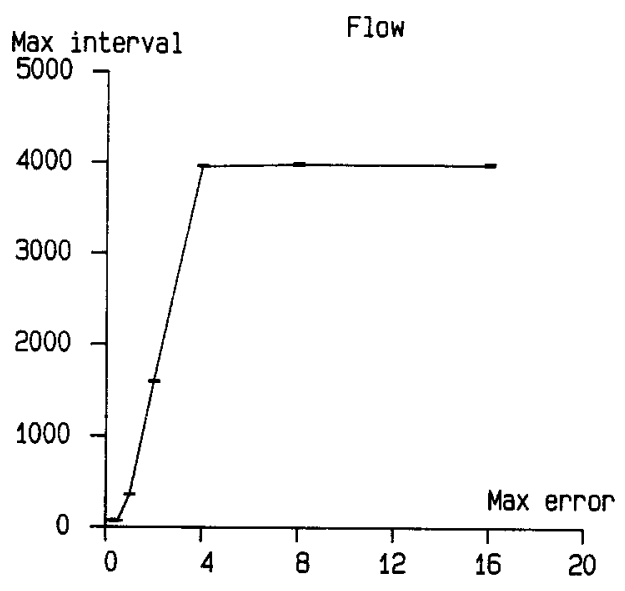

Fig. 8. The maximum number of contiguous samples discarded in each flow measurement as a function of maximum permitted error. Average plus and minus standard error of the mean are shown

compression technique as depicted in Figs. 3 and 6. Figure 6 shows a rapid decrease in the number of stored nonredundant samples at very small error values, representing the spontaneous base line fluctuation, followed by a much smaller decline at larger error values caused by the actual deletion of samples in the part of the signal where the patient was voiding. This interpretation is confirmed in Fig. 8 which shows a clear plateau representing the no-flow part of the recording. In contrast to Fig. 6, Fig. 3 shows a more gradual decrease in the number of permanent samples, as more and more signal variations are considered nonrelevant.

It can be concluded that the fan is a very good method indeed for reducing the number of samples to be permanently stored for the detrusor pressure signal. The actual maximum error allowed should be chosen on the basis of the noise level and digitizer sensitivity characteristic of the particular set of instrumentation, and the object of storing the data. Since all these were variable (for example, the noise in the pressure signal depends greatly on whether external or catheter-tip transducers are used) it is advisable to implement an error level that can be set by the user.

If the purpose had been to store the pressure signal for detailed future analysis, an error level of the order of 16 integer units or approximately $1.6 \mathrm{~cm} \mathrm{H}_{2} \mathrm{O}$ would have been suitable for the urodynamic apparatus with which the results in this study were obtained. This yields an $80 \%$ reduction in the number of samples to be stored (see Fig. 3), while the reconstructed signal resembles the original signal in great detail (see Fig. 2). The average absolute error at this maximum error value is approximately 5 integer units, or $0.5 \mathrm{~cm} \mathrm{H}_{2} \mathrm{O}$, which seems quite accurate enough given the size of the 

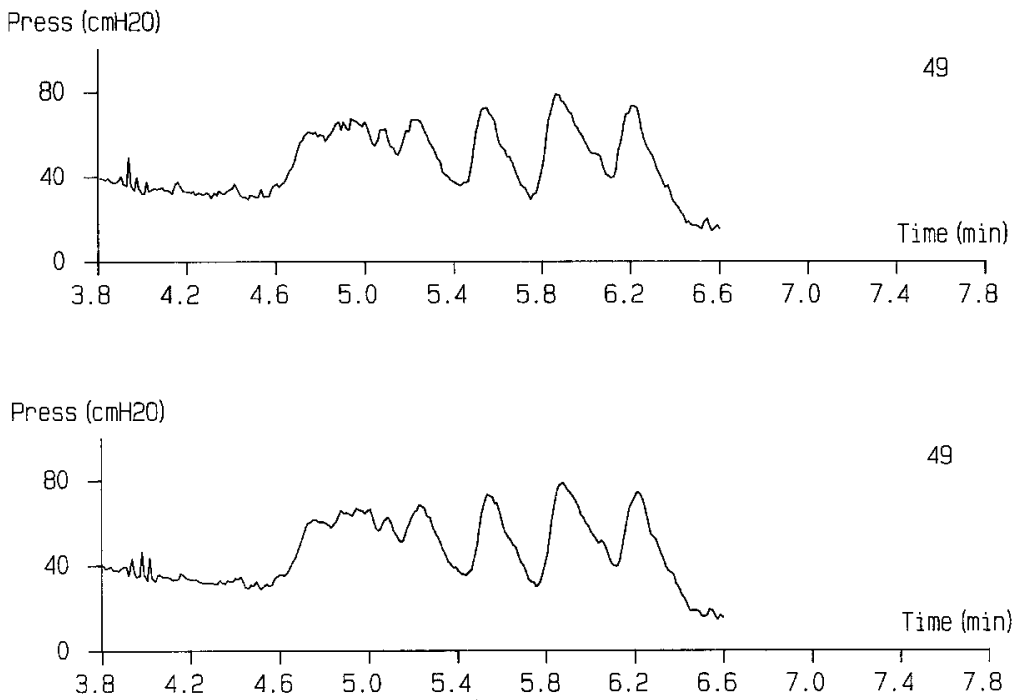

Fig. 9. The original data of Fig. 2 resampled at a uniform rate by taking every sixth sample, so as to yield 280 permanent samples
Fig. 10. The original data of Fig. 2 reduced to 280 permanent samples by averaging each set of six consecutive samples

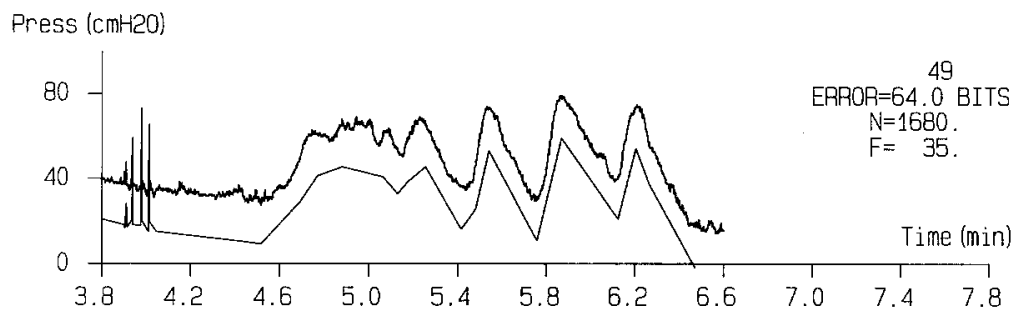

Fig. 11. Further reduction of the data in Fig. 2 to 35 permanent samples. Upper trace shows original data, lower trace reconstructed data errors to be expected in pressure measurements. The number of samples stored permanently in this case is equal to the number that would have been stored if the signal had been sampled at a continuous rate of 2 samples per second. Figure 9 shows the result of resampling the data in Fig. 2, taking every sixth sample so as to yield approximately the same number of permanent samples (280 as compared to 258 in Fig. 2). Notice the deformation of the four cough peaks in Fig. 9 as compared to Fig. 2. Figure 10 shows the original data, averaged to yield the same number of permanent data points (280). Again the cough peaks are deformed, showing the superiority of fan compressed data above uniformly sampled or averaged data.

Another purpose of compressing pressure data could be to keep a minimal record for future reference, in which case a much greater compression can be applied. Figure 11 shows the data of Fig. 2 reduced to $2 \%$ of the original number of samples by allowing a maximum error of 64 integer units or $6.4 \mathrm{~cm} \mathrm{H}_{2} \mathrm{O}$, which results in an average absolute error of $2.0 \mathrm{~cm}$ $\mathrm{H}_{2} \mathrm{O}$ according to Fig. 4. The major features of the signal, including the four cough peaks, can still be recognized in Fig. 11 showing that it might be sufficient to store on average one sample for $5 \mathrm{~s}$ of data.
A drawback of any adaptive sampling method is that the compressed data is no longer uniformly sampled. Therefore, in order to be able to reconstruct the data, the time corresponding to each stored sample must also be stored. This increases the amount of stored data by a factor of two. In highly compressed data as in Fig. 11 this does not seem to be a real problem. In signals stored in more detail, as in Fig. 2, an alternative might be formed by not storing the time with each sample, but storing the number of missing samples between each pair of successive samples. If the number of missing samples is restricted to 16 , it may be stored in an efficient way by using the four unused bits in every stored 16 bit computer word since only 12 bits are required for the analog data. In Figs 3-5 the broken lines show the consequences of limiting the interval between samples to 16. Figure 3 shows that at small maximum errors there is hardly any decrease in the efficiency of the data compression, whereas Fig. 4 indicates that the average error corresponding to a given maximum error decreases somewhat, as is to be expected since more samples are stored.

In the digital storage of flow curves, the long intervals in which no voiding takes place has led to the widespread use of trigger techniques which guarantee 
that the flow signal is only stored if a certain trigger level is exceeded. If there is a series of small leaks over a long period, or an intermittent flow pattern, this can cause considerable difficulty. Therefore the fan data compression technique should be considered superior for flow recordings too. As the signal can be compressed easily to less than $10 \%$ of the original number of samples by omitting the empty signal stretches at a very low maximum error of 1 integer unit, or approximately $0.025 \mathrm{ml} / \mathrm{s}$ in the present configuration, this should guarantee modest storage requirements. Of course the stored signal can be compressed further, as shown for pressure signals, if desired. Whatever the degree of compression, the separate storage of the time value with each sample must be recommended since restricting the maximum interval between stored samples to 16 samples would cause to many samples to be stored during the no flow periods.

For both pressure and flowrate signals the fan is a very good and flexible method of data compression. In the present study the fan algorithm was applied retrospectively to compress stored curves. In practice the algorithm may be integrated with the sampling procedure, so that compressed data is stored directly. An adjustable error level would allow the urodynamicist to find his own compromise between storage demands and accuracy over a very wide range, of variable requirements.

\section{References}

1. Blanchard SM Barr RC (1985) Comparison of methods for adaptive sampling of cardiac electrograms and electrocardiograms. Med Biol Eng Comp 23:377-386

2. DiPersio DA, Barr RC (1985) Evaluation of the fan method of adaptive sampling on human electrocardiograms. Med Biol Eng Comp 23:401-410

3. Gardenhire LW (1964) Redundancy reduction, the key to adaptive telemetry. Proceedings of the Nat, Telemetry Conference, Los Angeles, Sections 1-5, 1-16

4. Gardenhire LW (1965) Data redundancy reduction for biomedical telemetry. In: Caceres Ca (ed) Biomedical telemetry, vol 11. Academic Press, New York, pp 255-298

5. Mastrigt $R$ van (1984) A computer program for on-line measurement, storage, analysis and retrieval of urodynamic data. Comp Prog Biomed 18:109-117

6. Mastrigt $R$ van (1987) Urodynamic analysis using an on-line computer. Neurourol Urodyn 6-3:206-207

7. Pollard AE, Barr RC (1987) Adaptive sampling of intracellular and extracellular cardiac potentials with the fan method. Med Biol Eng Comp 25:261-268

8. Regnier $\mathrm{CH}$ (1986) Current and future applications of computers in urodynamics. Neurourol Urodyn 5:343-371

R. van Mastrigt, $\mathrm{PhD}$

Department of Urology

Erasmus University

P.O. Box 1738

3000 DR Rotterdam

The Netherlands 